\title{
Post-Marketing Surveillance of the Safety Profile of lodixanol in the Outpatient CT Setting: A Prospective, Multicenter, Observational Study of Patient Risk Factors, Adverse Reactions and Preventive Measures in 9953 Patients
}

\author{
CT-Untersuchungen mit lodixanol in der klinischen ambulanten Routine. Eine \\ prospektive, Multizenterstudie zur Risikoabschätzung von Nebenwirkungen \\ und Präventivmaßnahmen bei 9953 Patienten
}

Author

Affiliation

\author{
F. H. H. Müller
}

Radiology \& Nuclear Medicine Center, Ludwigshafen

\author{
Key words \\ - CT \\ - contrast agents \\ - drugs/reactions \\ - iodixanol \\ post-marketing surveillance \\ - safety profile
}

received 26.5.2013

accepted 5.3.2014

Bibliography

DOI http://dx.doi.org/

10.1055/s-0034-1366370

Published online: 11.4.2014

Fortschr Röntgenstr 2014; 186 :

1028-1034 @ Georg Thieme

Verlag KG Stuttgart · New York . ISSN 1438-9029

\section{Correspondence}

Dr. Frank Hugo Heinz Müller

Radiology \& Nuclear Medicine Center

Otto-Stabel-Str. 2-4

67059 Ludwigshafen

Germany

Tel.: ++49/0171/8585783

Fax: ++49/06 21/510025

dr.frank.h.h.mueller@me.com

\section{Zusammenfassung}

$\nabla$

Ziel: Nicht-interventionelle Studie bei ambulanten, kontrastmittelunterstützten CT-Untersuchungen zur

1. Häufigkeitsabschätzung präventiver Maßnahmen zur Risikoreduktion von Nebenwirkungen

2. prospektive Erfassung von Nebenwirkungen (Häufigkeit und Schweregrad) nach Gabe des isoosmolaren Röntgenkontrastmittels Iodixanol

3. Abschätzung des Einflusses präventiver Maßnahmen auf Häufigkeit und Schwere der beobachteten Nebenwirkungen

Material und Methoden: Ausgewertet wurden die Angaben zu 9953 Patienten aus 66 radiologischen Zentren/Praxen in Deutschland. Patientencharakteristika und Applikationsparameter von Iodixanol sowie Reaktionen mit möglichem ursächlichen $\mathrm{Zu}$ sammenhang mit Iodixanol wurden durch standardisierte Patientenbögen erfasst und bis zu sieben Tage nach Kontrastmittelapplikation ausgewertet.

Ergebnisse: Insgesamt zeigten 55,5\% der Patienten einen oder mehrere Risikofaktoren (z. B. 4,4\% eingeschränkte Nierenfunktion, 8,5\% Diabetes mellitus, 20,6\% erhöhten Blutdruck). Ein Drittel der Praxen setzte keinerlei präventive Maßnahmen ein. Patienten mit bekanntem Risiko für allergoide Reaktionen wurden häufiger medikamentös prämediziert (0,5-50,5\%). Häufigste präventive Maßnahme bei Patienten mit renalen Risikofaktoren war die orale $(<8 \%)$, gefolgt von der intravenösen Hydrierung (1\%). Nebenwirkungen, überwiegend Hypersensitivitätsreaktionen, wurden für 77 Patienten berichtet $(0,74 \%)$, davon bei 3 Patienten $(0,03 \%)$ als schwerwiegend eingestuft.

Schlussfolgerung: Der Einsatz präventiver Maßnahmen bei ambulanten CT-Untersuchungen war insgesamt gering, bei Risikopatienten je nach Anamnese etwas häufiger. In der klinischen Routine bei ambulanten CT-Untersuchungen zeigte sich das isoosmolare Iodixanol bei annähernd 10000 Patienten als sehr verträglich. Es konnte keine Kor-

\section{Abstract \\ $\nabla$}

Purpose: Non-interventional study in outpatient, contrast-enhanced CT

1. to determine the extent of preventive measures for risk reduction of adverse drug reactions after contrast-enhanced CT examinations.

2. to prospectively determine the incidence and severity of adverse drug reactions occurring after administration of the iso-osmolar contrast medium iodixanol

3. to determine a possible influence of preventive measures on the incidence/severity of adverse drug reactions.

Materials and Methods: Evaluable documentation was provided for 9953 patients from 66 radiology centers across Germany. Patient characteristics, aspects of iodixanol administration, and adverse events with an at least "possible" relationship were documented on a standardized case report form (CRF) and were evaluated up to seven days after contrast medium administration.

Results: About $55.5 \%$ of patients showed one or more risk factors (e.g. impaired renal function $4.4 \%$, diabetes mellitus $8.5 \%$, hypertension $20.6 \%$ ). One third of the sites did not implement any preventive measures. Patients with a known risk for an allergy-like reaction were more likely to receive pharmacologic preventive treatment $(0.5-50.5 \%)$. Oral hydration was the main preventive measure in patients with renal risk factors $(<8 \%)$ followed by intravenous hydration ( $1 \%)$. Adverse drug reactions, mainly hypersensitivity reactions, occurred in 77 patients $(0.74 \%)$, but were classified as serious in only 3 patients $(0.03 \%)$. No statistically significant correlation between risk factors, preventive measures, and adverse reactions could be found.

Conclusion: The use of preventive measures for CT examinations in this outpatient setting was generally low with risk patients being pre-medicated 
relation zwischen Risikofaktoren, Präventivmaßnahmen sowie der Häufigkeit von Nebenwirkungen gefunden werden.

Kernaussagen:

- Präventivmaßnahmen selten bei ambulanten CT-Untersuchungen

- Geringe Rate an akuten und verzögerten Nebenwirkungen nach Iodixanol

- Kein erkennbarer Zusammenhang zwischen Risikofaktoren, Präventivmassnahmen und Nebenwirkungen more often, depending on their history. In the routine outpatient setting, iso-osmolar iodixanol was very well tolerated in almost 10000 patients undergoing diagnostic CT. The rate of acute and delayed adverse reactions was low. No correlation could be found between risk factors, preventive measures and the incidence of adverse drug reactions.

Key Points:

- Rare use of preventive measures for outpatient CT examinations.

- Low rate of acute and late adverse drug reactions to iodixanol.

- No correlation between risk factors, preventive measures and adverse drug reactions.

Citation Format:

- Müller FHH. Post-Marketing Surveillance of the Safety Profile of Iodixanol in the Outpatient CT Setting: A Prospective, Multicenter, Observational Study of Patient Risk Factors, Adverse Reactions and Preventive Measures in 9953 Patients. Fortschr Röntgenstr 2014; 186: 1028-1034

\section{Background}

$\nabla$

Adverse drug reactions associated with the administration of iodinated contrast media (CM) are generally rare and mild in nature, but severe and even life-threatening reactions may occur. Much controversy surrounds the issue of preventive measures to reduce either allergy-like reactions or renal reactions in the outpatient setting. For the prevention of allergy-like reactions, pre-treatment with steroids (and antihistamines) seems to be the preferred method, as described in a survey done by the European Society of Urogenital Radiology (ESUR) in 2001. However, the issue is still under debate, as even the ESUR, which recommends pre-medication in patients at high risk for allergylike reactions, has stated that clinical evidence for the usefulness of preventive measures is limited [1]. Different studies have established that (intravenous) hydration prior to and after CM administration has beneficial effects on the incidence of contrast-induced acute kidney injury (CI-AKI), previously known as contrast-induced nephropathy (CIN) [2-4]. Most of these studies were set in a clinical environment and were controlled clinical trials where the use of preventive measures was predefined and not left to the discretion of the treating physician. However, there is general consensus that hydration is the most important measure to prevent CI-AKI [1]. There are only a few randomized controlled studies comparing the CI-AKI risk after oral and intravenous hydration with inconsistent results $[5,6]$. A current meta-analysis found no significant difference between pre-procedural intravenous and oral volume expansion for the prevention of CI-AKI [7].

As computed tomography (CT) becomes more readily available and technology continues to broaden the potential applications for $\mathrm{CT}$, the number of $\mathrm{CT}$ scans performed worldwide each year will continue to increase $[8,9]$. It therefore becomes increasingly important to seek additional information about current preventive measures and the extent to which they are used, especially in the outpatient setting.

This Non-Interventional Study (NIS) was specifically designed to investigate this issue. It is intended to provide information about the use of preventive measures, especially in the context of patient risk profiles and their potential impact on the incidence of adverse drug reactions. It is intended to give further insight regarding the extent to which preventive measures are used, for what type of patients (all patients, or only certain risk groups), and which types of preventive measures are most commonly used.

\section{Material and Methods \\ $\nabla$}

The NIS was performed as a prospective, non-randomized, multicenter, open-label study in 66 German radiology centers. Data from outpatients receiving a diagnostic CM-enhanced CT with administration of iso-osmolar iodixanol in accordance with the Summary of Product Characteristics were documented on standardized case report forms (CRF). The sites were asked to document subsequently all patients scheduled for a CT examination with iodixanol. Patients were to be included only once, even if they had several CT scans during the recruitment period of the NIS. Patient recruitment lasted from June 2009 to January 2010. The German Federal Institute for Drugs and Medical Devices (BfArM), the National Associations of Panel Doctors (KVB) and the representative body of the Statutory Sickness Funds were notified of the performance of this study.

To harmonize the documentation of risk factors, the standardized CRF included a predefined list of potential risk factors (risk of adverse events, renal risk factors) based on the ESUR definitions in 2009. CRF parameters included: patient demographics (age, gender), medical data (risk profile [based on physician's assessment]: previous allergy-like reaction to a $\mathrm{CM}$, allergies requiring treatment, asthma, heart failure, diabetes requiring treatment, renal impairment, kidney surgery, proteinuria, hypertension, gout, intake of nephrotoxic drugs, and clinical signs of dehydration), region of investigation (head/neck, chest/lung, abdomen/ pelvis, upper extremities, lower extremities, $\bullet$ Table 1), contrast medium used (iodixanol $270 \mathrm{mgl} / \mathrm{ml}$, iodixanol $320 \mathrm{mgl} / \mathrm{ml}$ [VISIPAQUE ${ }^{\mathrm{TM}}$, GE Healthcare]), mode of injection (manual versus power injection), flow rate, total $\mathrm{CM}$ volume, occurrence of adverse reactions (acute or late), and preventive measures taken. Any preventive measures taken were recorded. The measures were categorized as follows: oral hydration, i. v. hydration withdrawal of concomitant medication, initiation of pharmacological measures to prevent CI-AKI, premedication. Due to the non-interventional character of the NIS, only patient data being part of 
Table 1 Region of investigation.

Tab. 1 Untersuchte Körperregionen.

\begin{tabular}{|l|l|}
$\begin{array}{l}\text { region of CT } \\
\text { (by frequency) }\end{array}$ & $\begin{array}{l}\text { number of patients } \\
\mathbf{n}(\%)\end{array}$ \\
\hline abdomen/pelvis & $4909(49.3 \%)$ \\
\hline thorax/lung & $3314(33.3 \%)$ \\
\hline head/neck & $2539(25.5 \%)$ \\
\hline lower extremities & $41(0.4 \%)$ \\
\hline upper extremities & $25(0.3 \%)$ \\
\hline
\end{tabular}

${ }^{1}$ More than one answer possible, ratios and percentages based on available and evaluable data.

Mehr als eine Option möglich, Zahlen und Prozentangaben basieren auf verfügbaren und berechenbaren Daten.

their physician's daily practice routine were documented. No additional investigations were performed.

On an additional site questionnaire, every center documented once the type of CT scanner (number of rows), the CM temperature at injection, the type of injection (manual versus power injector), and the type of fluid used for i.v. hydration (saline, half saline, or sodium bicarbonate).

Recorded adverse events were classified into acute reactions occurring within one hour of CM administration (while the patients were still on site), and late reactions, occurring from 1 hour up to one week after CM administration. The investigators had been instructed to request their patients to report any adverse events occurring up to seven days after $\mathrm{CM}$ administration. These late adverse events were documented on a separate AE report form.

The sample size calculation was based on the results of a previous NIS [10]. The sample size calculation resulted in a total of 12000 patients needed to obtain a sufficient number of patients in each subgroup (risk patients for allergy-like or renal reactions), taking into account an estimated dropout rate of $5 \%$.

Data entry was carried out according to guidelines including double data entry and checks for plausibility and consistency. Implausible data in the database were either corrected, if appropriate, or queried with the radiologist for correction or confirmation. If there was no confirmation or correction from the radiologists, this information was recorded as "missing" and was not included in the analyses. Adverse drug reactions (ADRs) were coded according to MedDRA version 11.0. Data were analyzed using descriptive statistics, with SAS software version 9.1. The Chi-square test was used to test for differences in acute and late reactions in relation to preventive measures and risk factors. As a certain amount of missing data is inevitable in NIS studies, all ratios/percentages are based on available and evaluable data.

\section{Results}

$\nabla$

\section{Surveillance population}

66 radiologists in private clinic-based (non-hospital) practice contributed to this study. The NIS started on June 1, 2009 and was terminated on January 13, 2010 with the closure of the NIS database. In this period 9953 patients had been documented. 401 CRFs arrived after closure of the study database and were not included in the treatment analysis population. Nevertheless, these "late arrivals" were included in the safety population, which thus included a total of 10354 patients.
Table 2 Risk factors. ${ }^{1}$

Tab. 2 Risikofaktoren. ${ }^{2}$

\begin{tabular}{ll}
\hline risk factor (by frequency) & number of patients (\%) \\
\hline \begin{tabular}{l} 
age > 70 years \\
$\begin{array}{l}\text { hypertension } \\
\text { (risk factor for elevated SCr) }\end{array}$ \\
\hline diabetes mellitus requiring treatment
\end{tabular} & $3119(31.3 \%)$ \\
\hline cardiac insufficiency & $845(8.5 \%)$ \\
\hline renal insufficiency & $553(5.6 \%)$ \\
\hline asthma & $435(4.4 \%)$ \\
\hline allergy requiring treatment & $379(3.8 \%)$ \\
\hline prior renal surgery & $272(2.7 \%)$ \\
\hline $\begin{array}{l}\text { nephrotoxic medication } \\
\text { (regular intake) }\end{array}$ & $253(2.5 \%)$ \\
\hline gout & $251(2.5 \%)$ \\
\hline prior allergy-like reaction to CM & $207(2.1 \%)$ \\
\hline dehydration & $97(1.0 \%)$ \\
\hline $\begin{array}{l}\text { proteinuria } \\
\text { (risk factor for elevated SCr) }\end{array}$ & $87(0.9 \%)$ \\
\hline
\end{tabular}

${ }^{1}$ As assessed by the radiologist.

2 Eingeschätzt durch den Radiologen.

More than half of the patients had at least one known risk factor (55.5\%) that suggested they might be at higher risk for allergylike or renal adverse reactions. Patient demographics and procedural information are given in $\bullet$ Table 2.

The individual assessment of patient risk factors was done by the physician performing the examination (as mandatory for NIS, without any additional testing). The group of renal risk patients included 435 patients (4.4\%), for whom a more recent $\mathrm{SCr}$ was available to the physician in $89.7 \%$ (390/435) of patients. Whereas the CRF asked for SCr values only, physicians supplied additional eGFR values for another 133 patients. 12 patients had an eGFR $<45 \mathrm{ml} / \mathrm{min} / 1.73 \mathrm{~m}^{2}, 76$ patients $<60 \mathrm{ml} / \mathrm{min} / 1.73 \mathrm{~m}^{2}$, with the mean eGFR being $60.6 \mathrm{ml} / \mathrm{min} / 1.73 \mathrm{~m}^{2}$. As this was a non-interventional study, no post-CM administration SCr values were measured/recorded since this is not part of the routine in the outpatient setting.

\section{Contrast medium administration}

$60 \%$ of the patient population received the iso-osmolar CM iodixanol $270 \mathrm{mgl} / \mathrm{ml}, 40 \%$ received the iso-osmolar CM iodixanol $320 \mathrm{mgl} / \mathrm{ml}$ (subsequently referred to as iodixanol 270 and iodixanol 320). 40 sites used iodixanol 270 exclusively, 19 sites used iodixanol 320 exclusively. Only 7 sites used both concentrations. The total administered CM volume was reported for 9933 patients, with a median of $100 \mathrm{ml}$. The mean volume did not differ significantly between iodixanol 270 and iodixanol 320, 0 Table 3.

\section{Safety}

77 patients ( $0.74 \%$ of all patients) from 29 sites experienced adverse reactions (ADR). Reactions of 53 patients ( $0.51 \%$ ) were classified as hypersensitivity reactions. 19 patients $(0.18 \%)$ experienced nausea immediately after CM administration. Only one of the patients with a prior allergy-like reaction to CM experienced an allergy-like reaction in this study (non-serious hypersensitivity reaction, no preventive measures). $\bullet$ Table 4 shows the details of all ADRs.

Three adverse reactions were classified as serious $(0.03 \%$ of all patients). All three occurred while the patients were still on-site. 
Table 3 Demographics and procedural information. ${ }^{1}$

Tab. 3 Demografische Information und Details zur Applikation. ${ }^{2}$

\begin{tabular}{|llc|}
$\begin{array}{l}\text { gender } \\
\text { (\# of patients) }\end{array}$ & male & 5056 \\
\hline $\begin{array}{l}\text { age (years) } \\
(n=9949)\end{array}$ & $\begin{array}{l}\text { mean/SD } \\
\text { iodine concentration }\end{array}$ & 4893 \\
\hline & $(\mathrm{mgl} / \mathrm{ml})$ & (\# of patients) \\
\hline & 270 & 5956 \\
\hline total volume $(\mathrm{ml})$ & 320 & 3977 \\
$(n=9933)$ & mean/SD & $92.5 / 23.08$ \\
\hline
\end{tabular}

${ }^{1}$ Ratios and percentages based on available and evaluable data.

2 Zahlen und Prozentangaben basieren auf verfügbaren und berechenbaren Daten.

Table 4 Characteristics and incidence of adverse drug reactions (ADRs) reported by $>1$ patient.

Tab. 4 Art und Häufigkeit akuter und verzögerter Nebenwirkungen (ADR), falls mehr als bei 1 Patienten beschrieben.

\begin{tabular}{|c|c|}
\hline & $\begin{array}{l}\text { no. of patients } \\
\text { (\%)/no. of events }\end{array}$ \\
\hline SOC/preferred term & $(\mathrm{N}=10354)$ \\
\hline any system organ class ${ }^{1}$ & $77(0.74) 208$ \\
\hline skin and subcutaneous tissue disorders & $54(0.52) 89$ \\
\hline pruritus & $22(0.21) 22$ \\
\hline erythema & $17(0.16) 17$ \\
\hline rash & $13(0.13) 13$ \\
\hline urticaria & $12(0.12) 12$ \\
\hline swelling face & $4(0.04) 4$ \\
\hline generalized erythema & $3(0.03) 3$ \\
\hline rash macular & $3(0.03) 3$ \\
\hline rash generalized & $2(0.02) 2$ \\
\hline skin burning sensation & $2(0.02) 2$ \\
\hline urticaria & $2(0.02) 2$ \\
\hline immune system disorder & $57(0.55) 57$ \\
\hline hypersensitivity & $53(0.51) 53$ \\
\hline anaphylactoid reaction & $3(0.03) 3$ \\
\hline anaphylactoid shock & $1(0.01) 1$ \\
\hline gastrointestinal disorder & $22(0.21) 26$ \\
\hline nausea & $19(0.18) 19$ \\
\hline vomiting & $5(0.05) 5$ \\
\hline general disorders and administration site condition & $12(0.12) 13$ \\
\hline feeling hot & $4(0.04) 4$ \\
\hline swelling & $3(0.03) 3$ \\
\hline sensation of foreign body & $2(0.02) 2$ \\
\hline infections and infestations & $6(0.06) 6$ \\
\hline rash pustular & $6(0.06) 6$ \\
\hline respiratory thoracic and mediastinal disorder & $5(0.05) 5$ \\
\hline throat irritations & $3(0.03) 3$ \\
\hline sneezing & $2(0.02) 2$ \\
\hline eye disorders & $3(0.03) 3$ \\
\hline eyelid edema & $2(0.02) 2$ \\
\hline nervous system disorder & $3(0.03) 3$ \\
\hline cardiac disorder & $2(0.02) 2$ \\
\hline investigations & $2(0.02) 2$ \\
\hline blood pressure decreased & $2(0.02) 2$ \\
\hline
\end{tabular}

${ }^{1}$ ADRs experienced by only one patient are not detailed (exception: anaphylactoid shock is listed, as this was a serious reaction).

Nebenwirkungen, die nur einmalig auftraten wurden nicht aufgeführt (Ausnahme: Anaphylaktischer Schock als schwere unerwünschte Nebenwirkung).
Table 5 Serious adverse drug reactions.

Tab. 5 Schwerwiegende Nebenwirkungen.

\begin{tabular}{llll} 
gender & female & female & female \\
\hline age $^{1}$ [years] & 41 & 62 & 60 \\
\hline latency & acute & acute & acute \\
\hline $\begin{array}{l}\text { prior reaction } \\
\text { to CM }\end{array}$ & no data available & no data available & no \\
\hline $\begin{array}{l}\text { SADR } \\
\text { as summarized } \\
\text { (PT) }\end{array}$ & $\begin{array}{l}\text { anaphylactoid } \\
\text { shock } \\
\text { (shock, loss of } \\
\text { consciousness, } \\
\text { anaphylactoid } \\
\text { shock) }\end{array}$ & $\begin{array}{l}\text { anaphylactoid } \\
\text { reaction } \\
\text { (not further } \\
\text { specified by } \\
\text { the physician) }\end{array}$ & $\begin{array}{l}\text { hyper-sensitivity } \\
\text { (hypersensitivity, } \\
\text { throat irritation, } \\
\text { paraesthesia oral) }\end{array}$ \\
\hline recovered & recovered & recovered \\
\hline
\end{tabular}

${ }^{1}$ At time of event.

Zum Zeitpunkt der Untersuchung.

One patient was hospitalized for observation due to an anaphylactic reaction, which lasted for 75 minutes. The second patient experienced a hypersensitivity reaction and was therefore hospitalized overnight for observation. The third patient was classified as serious due to the severity of the reaction. All three patients recovered fully. Table 5 shows the overview of the serious ADRs.

\section{Acute and late reactions}

Acute reactions occurred in 35 patients $(0.34 \%)$, with a median latency of 44 minutes. Acute reactions were mainly hypersensitivity reactions, including skin and/or subcutaneous reactions $(n=29)$, and gastrointestinal disorders $(n=20)$, mostly nausea directly after CM application $(\mathrm{n}=18)$.

40 patients $(0.39 \%)$ experienced late reactions. All 40 patients had skin/subcutaneous reactions with the majority of them coded as hypersensitivity reactions (39 patients). $48.5 \%$ of the late adverse reactions required treatment, mostly in the form of antihistamines/antiallergics being provided by general practitioners (56.25\%), medical specialists (37.5\%), or the hospital (6.25\%). No late renal reactions were reported.

In two patients, latency was unknown due to missing data.

\section{Preventive measures}

The following preventive measures were recorded: oral/i.v. hydration, before/after CM administration, withdrawal of drugs (e. g. biguanides, NSAIDs), initiation of pharmacological measures to prevent CI-AKI (e.g. NAC) or specific premedication to prevent allergy-like reactions (e.g. steroids, antihistamines).

\section{Preventive measures by participating sites}

Of the 66 sites, about 1/3 did not apply any preventive measures, regardless of their patients' risk profile. 29 (44\%) of the 66 sites hydrated part of their patients either orally $(8.2 \%$; 814$)$ or i.v. (1\%; 97 patients). Two sites hydrated all patients (one site only orally, one site only i.v.) regardless of their risk profile for CI-AKI. Only 3 centers documented using either sodium bicarbonate or half-normal saline $(0.45 \% \mathrm{NaCl})$ for i.v. hydration, while all others used normal saline $(0.9 \% \mathrm{NaCl})$. 
Table 6 Preventive measures.

Tab. 6 Präventive Maßnahmen.

\begin{tabular}{|c|c|c|c|c|}
\hline \multirow[t]{2}{*}{ risk factor } & \multicolumn{4}{|c|}{$\begin{array}{l}\text { patients receiving preventive measures } \\
\% \text { of patients with that risk factor| } \\
\% \text { patients without that risk factor }\end{array}$} \\
\hline & $\begin{array}{l}\text { oral } \\
\text { hydration }\end{array}$ & $\begin{array}{l}\text { i.v. } \\
\text { hydration }\end{array}$ & $\begin{array}{l}\text { NSAID treatment } \\
\text { stopped }\end{array}$ & $\begin{array}{l}\text { treatment } \\
\text { with NAC }\end{array}$ \\
\hline impaired renal & 21.5 & 6.0 & 0.0 & 1.4 \\
\hline $\begin{array}{l}\text { function } \\
(n=435)\end{array}$ & 7.8 & 0.6 & 0.1 & 0.0 \\
\hline previous kidney & 11.9 & 3.6 & 0.0 & 0.0 \\
\hline surgery $(n=253)$ & 8.1 & 0.8 & 0.1 & 0.1 \\
\hline diabetes mellitus & 10.9 & 2.2 & 0.4 & 0.2 \\
\hline$(n=845)$ & 7.9 & 0.8 & 0.0 & 0.1 \\
\hline proteinuria & 75.0 & 16.7 & 0.0 & 0.0 \\
\hline$(n=12)$ & 5.5 & 0.8 & 0.1 & 0.0 \\
\hline regular intake & 14.3 & 5.2 & 2.4 & 0.4 \\
\hline $\begin{array}{l}\text { of nephrotoxic } \\
\text { medication } \\
(n=251)\end{array}$ & 8.5 & 0.8 & 0.0 & 0.1 \\
\hline dehydration & 34.5 & 17.2 & 1.1 & 0.0 \\
\hline$(n=87)$ & 7.9 & 0.8 & 0.1 & 0.1 \\
\hline
\end{tabular}

\section{Relationship between risk factors}

and preventive measures

Risk for allergy-like reactions

Patients with a higher risk for allergy-like reactions had been defined as patients with allergies requiring treatment, asthma, or a previous allergy-like reaction to $\mathrm{CM}$. Of the 272 patients with documented allergies, $0.7 \%$ received steroids and $4.8 \%$ received antihistamines prior to $\mathrm{CM}$ administration. Of the patients with asthma ( $\mathrm{n}=379), 0.5 \%$ received steroids and $0.8 \%$ antihistamines. Patients without those risk factors received steroids and antihistamines in comparable ratios. Only patients with a history of allergy-like reaction to $\mathrm{CM}$ were pretreated significantly more often. In this group of 97 patients, $21.6 \%$ received steroids and $50.5 \%$ antihistamines. Among patients without this risk factor, hardly any received steroids or antihistamines $(0.0 \%$ and $0.2 \%$, respectively).

\section{Risk for $\mathrm{Cl}-\mathrm{AKI}$}

Patients with a higher risk for CI-AKI were defined as patients with impaired renal function, previous kidney surgery, regular intake of nephrotoxic medication, proteinuria, diabetes mellitus, and signs of dehydration.

The NIS identified that appropriate preventive measures (hydration, withdrawal of nephrotoxic medication, treatment with NAC) were used more frequently in these risk patients, mainly in the form of either oral or i. v. hydration. $\bullet$ Table 6 shows the different pre-treatments in relation to the presence of renal risk factors.

\section{Relationship between adverse drug reactions, preven-} tive measures and risk factors

The overall incidence of allergy-like adverse drug reactions in patients with one or more risk factors for such reactions was $0.69 \%$ compared to $0.63 \%$ in patients without any risk factors. Risk patients (history of allergy-like reaction to $\mathrm{CM}$ ) receiving preventive medication did not show a significantly lower rate of adverse events, compared to risk patients with no preventive medication
Table 7 Patients with adverse reactions by risk factors for acute/late reactions and preventive measures.

Tab. 7 Patienten mit Nebenwirkungen und Risikofaktoren für Akut- und Spätreaktionen im Vergleich zu Präventivmaßnahmen.

\begin{tabular}{|c|c|c|c|c|}
\hline \multicolumn{5}{|c|}{ patients with adverse reactions: $\mathbf{n}$ (\% patients) } \\
\hline & \multicolumn{2}{|c|}{ acute reaction } & \multicolumn{2}{|c|}{ late reaction } \\
\hline appropriate preventive measure ${ }^{1}$ ? & yes & no & yes & no \\
\hline \multicolumn{5}{|l|}{ risk factor } \\
\hline \multirow[t]{2}{*}{ previous allergy-like reaction } & 0 & 2 & 0 & 4 \\
\hline & $0.0 \%$ & $0.3 \%$ & $0.0 \%$ & $0.6 \%$ \\
\hline
\end{tabular}

(statistical testing [FISHERs exact test]: difference not significant). No renal adverse reactions were reported.

A logistic regression analysis failed to reveal any correlation between risk factors, preventive measures, and the incidence of adverse drug reactions. $\odot$ Table 7 shows the adverse reactions in relation to preventive measures for patients with risk factors for acute/late reactions.

\section{Discussion}

$\nabla$

Adverse drug reactions (ADRs) associated with the administration of iodinated contrast media (CM) are generally rare and mild in nature, but severe and even life-threatening reactions may occur. Adverse reactions are grouped into acute reactions, occurring up to one hour after $\mathrm{CM}$ administration, and late reactions, occurring from 1 hour up to one week after exposure to CM [11]. Acute reactions often present as allergy-like or anaphylactoid reactions and range in severity from mild symptoms like urticaria and itching to severe reactions such as cardiopulmonary arrest and death $(<1 /$ 100000 patients) $[12,13]$. The majority of late reactions are mild to moderate skin reactions which are considered as late hypersensitivity reactions [14]. Risk factors for both acute and late reactions include previous adverse reactions (previous late reactions predispose for late reactions and previous immediate reactions for immediate reactions) to CM, a history of allergy, or asthma [14, 15]. Risk factors for CI-AKI include age, congestive heart failure, use of nephrotoxic drugs, and chronic kidney disease, especially diabetic nephropathy $[1,16,17]$.

The present NIS aimed at evaluating the incidence of adverse drug reactions in relation to individual patient risk factors and preventive measures taken to prevent such ADRs. In this regard, radiologists from 66 outpatient radiology sites across Germany documented data of roughly 10000 patients undergoing CM-enhanced diagnostic CT with the non-ionic iso-osmolar contrast medium iodixanol.

Although the number of total adverse drug reactions is apparently low (77 patients, $0.74 \%$ ), the findings are in agreement with published studies on ADRs after CT scans [18 - 20]. In these studies adverse events after i.v. CM administration ranged between $0.2 \%$ and $3.1 \%$. Similar ADR rates were also observed in larger trials $[21,22]$. The number of allergy-like adverse drug reactions is comparable to other published NIS with non-ionic contrast media $[10,23]$. 
The incidence of serious adverse drug reactions was very low $(0.03 \%)$ and all SAE occurred in the radiology office allowing for immediate medical intervention. Importantly, no serious late reaction was reported which is in accordance with the literature describing severe late reactions to be very rare [14].

The incidences of both acute and late adverse reactions were low but consistent with a previous study $(0.34 \%$ and $0.39 \%$, as compared to $0.32 \%$ and $0.42 \%$ ) [10]. To record late adverse reactions, patients had been instructed by the physicians to report any adverse events occurring between one hour and seven days after CM administration. However, it is a limitation of this non-interventional trial that $\mathrm{AE}$ reporting relied on patient collaboration and thus some late events may not have been reported to the investigator. Hypersensitivity reactions were the most common reactions among both acute and late reactions, occurring in 53 of 77 patients.

As in a previous NIS [10], more than half of the patients had one or more of the documented risk factors. Even though $7.5 \%$ of all patients were considered to be at risk for an allergy- like reaction, only a rather small part of them received preventive treatment despite guideline recommendations $[10,15]$. Measures to prevent allergy-like reactions were applied very heterogeneously depending on the kind of risk factor: antihistamines were given in $50 \%$ versus $0.2 \%$ of the patients with/without previous allergy-like reaction in $4.8 \%$ vs. $0.5 \%$ of patients with/without allergies, and in $0.8 \%$ vs. $0.7 \%$ of patients with/without asthma. Steroid premedication was used in $22 \%$ of the patients with a former allergy-like reaction (21 of 97). As only one of the 97 patients with a former allergy-like reaction to a CM experienced a reaction during this study, no treatment effect was seen in terms of adverse event reduction. The data obtained within this NIS therefore do not suggest that patients with previous reactions to $\mathrm{CM}$ are at increased risk for experiencing another ADR after re-exposure to a CM. However, the overall low number of reactions limits the value of this statement which differs from the risk described in the literature and subsequent guideline recommendations.

Measures to prevent CI-AKI are recommended by the ESUR. Our study also aimed at determining whether preventive measures are commonly used in the outpatient setting. The data suggest that measures to prevent $\mathrm{CI}$-AKI were used very heterogeneously. One third of the sites did not use any preventive measures for patients at increased risk for $\mathrm{CI}-\mathrm{AKI}$, whereas others pre-treated all of their patients, regardless of their risk profile. Overall, the most common preventive measure was oral hydration, which was provided to $16.5 \%$ of patients with renal insufficiency/previous kidney surgery/proteinuria. In patients with clinical signs of dehydration, preventive measures were taken more often (34.5\%) although it is not clear whether hydration was used to protect the kidneys or to remedy the dehydration itself. In total, i. v. hydration was only given in $5.2 \%$ of the patients with the previously mentioned risk factors. Regarding the type of fluid used for i.v. hydration, results are in accordance with other studies indicating that normal saline is the preferred hydration agent rather than half-normal saline $(92.5 \%$ versus $5 \%$ of the sites using normal versus half normal saline) [3].

Discontinuation of nephrotoxic medication, although recommended by guidelines and SmPC for patients with renal insufficiency, was not done for patients with renal risk factors in this NIS $[24,25]$. The reason for this discrepancy could be the general structure of the outpatient setting system. With patients being transcribed by the general practitioner for diagnostic imaging, radiologists may not be aware of specific diseases or medications in order to be able to withdraw the respective drug before administering $\mathrm{CM}$.

The incidence of $\mathrm{CI}$-AKI could not be assessed in this NIS due to the unavailability of post-CM SCr values in the routine outpatient setting. In the absence of post- $\mathrm{SCr}$ values, the only possible conclusion in terms of possible renal function deterioration from this NIS is that obviously no acute kidney failure occurred, as this would probably have been reported as an adverse event to the investigator.

In summary, there was no statistically significant evidence to suggest a relationship between any of the risk factors, preventive measures and adverse drug reactions. The finding that preventive measures are rarely used in the outpatient setting except in certain high risk patients with e.g. former allergy-like reaction to a CM may be explained by two factors: physicians might not be aware of current recommendations in this regard or might not consider preventive measures useful except in highest risk patients.

\section{Conclusion \\ $\nabla$}

Preventive measures to reduce the risk of contrast medium-induced adverse drug reactions are not widely used in the outpatient setting, neither for the prevention of allergy-like reactions nor for the prevention of CI-AKI. The underlying reasons for this behavior have not been analyzed so far.

To assess the motivation for or against the use of preventive measures, additional studies should be performed, especially in the outpatient setting where few data are available regarding the daily routine due to the fact that most clinical trials are being performed in hospital settings. In summary, ADR incidence after the use of the iso-osmolar contrast agent iodixanol was very low (the incidence of acute and late adverse reactions was $0.34 \%$ and $0.39 \%$, respectively) with a very rare incidence of serious adverse reactions $(0.03 \%)$. Patient-related risk factors and preventive measures did not seem to influence the incidence of adverse reactions. However, final conclusions in this regard cannot be drawn, as the total number of events was too low to allow for proper statistical testing of the significance of the results and due to the limitations of this non-interventional study regarding the observational period. Although this study was not designed to compare the frequency of adverse events between various contrast media, the rare frequency of adverse events observed in a large outpatient population in this real-world CT setting indicates that iodixanol is a safe contrast medium in the outpatient setting even when recommended preventive measures are not applied.

\section{Clinical relevance of study:}

- Rare use of preventive measures for outpatient CT examinations.

Low rate of acute and late adverse drug reactions to iodixanol.

- No correlation between risk factors, preventive measures and adverse drug reactions.

- Iodinated radiographic contrast media (CM) are considered safe diagnostic agents with a low incidence of adverse drug reactions. 


\section{References:}

1 Stacul F. Contrast induced nephropathy: updated ESUR Contrast Media Safety Committee guidelines. Eur Radiol 2011; 21: 2527-2541

2 Weisbord SD, Palevsky PM. Preventions of contrast induced nephropathy with volume expansion. Clin J Am Soc Nephrol 2008; 3: $273-280$

3 Mueller C, Buerkle G, Buettner HJ et al. Prevention of contrast mediaassociated nephropathy: randomized comparison of 2 hydration regimens in 1620 patients undergoing coronary angioplasty. Arch Intern Med 2002; 162: 329-336

4 Reddan D, Laville M, Garovic VD. Contrast-induced nephropathy and its prevention: what do we really know from evidence-based findings? J Nephrol 2009; 22: 333-351

5 Trivedi HS, Moore H, Nasr S et al. A randomized prospective trial to assess the role of saline hydration on the development of contrast nephrotoxicity. Nephron Clin Pract 2003; 93: C29-C34

6 Taylor AJ, Hotchkiss D, Morse RW et al. PREPARED: Preparation for Angiography in Renal Dysfunction: a randomized trial of inpatient vs outpatient hydration protocols for cardiac catheterization in mild-tomoderate renal dysfunction. Chest 1998; 114: 1570 - 1574

7 Hiremath S et al. Prevention of Contrast-Induced Acute Kidney Injury: Is Simple Oral Hydration Similar To Intravenous? A Systematic Review of the Evidence. Plos One 2013, access 5th of Sept. 2013 via. http://www.plosone.org/article/info\%3Adoi\%2F10.1371\%2Fjournal. pone.0060009

8 Morcos SK, Thomsen HS, Webb JAW. Prevention of generalized reactions to contrast media: a consensus report and guidelines. Eur Radiol 2001; 11: $1720-1728$

9 Lasser EC, Berry CC, Lee B et al. Pre-treatment with corticosteroids to alleviate reactions to intravenous contrast material. N Engl J Med 1987; 317: 845-849

10 Häussler MD. Safety and patient comfort with iodixanol: A postmarketing surveillance study in 9515 patients undergoing diagnostic CT examinations. Acta Radiol 2010; 8: 924-933

11 Meth MJ, Maibach HI. Current understanding of contrast media reactions and implications for clinical management. Drug Safety 2006; 29 : $133-141$
12 Wang CL et al. Frequency, outcome, and appropriateness of treatment of nonionic iodinated contrast media reactions. Am J Roentgenol 2008; 191: 409-415

13 Caro JJ, Trindade E, McGregor M. The risks of death and of severe nonfatal reactions with high- vs low-osmolality contrast media: a metaanalysis. Am J Roentgenol 1991; 156: 825-832

14 Webb JAW, Stacul F, Thomsen HS et al. Late adverse reactions to intravascular iodinated contrast media. Eur Radiol 2003; 13: 181-184

15 Hosoya T, Yamaguchi K, Akutsu T et al. Delayed adverse reactions to iodinated contrast media and their risk factors. Radiat Med 2000; 18: $39-45$

16 Maddox TG. Adverse reactions to contrast material: recognition, prevention, and treatment. Am Fam Physician 2002; 66: 1229-1234

17 Davidson C, Stacul F, McCullogh PA et al. Contrast medium use. Am J Cardiol 2006; 98: 42K-58K

18 Mortelé K et al. Universal Use of Nonionic Iodinated Contrast Medium for CT: Evaluation of Safety in a Large Urban Teaching Hospital. Am J Roentgenol 2005; 185: 31 - 34

19 Trcka J et al. Anaphylaxis to Iodinated Contrast Material: Nonallergic Hypersensitivity or IgE-Mediated Allergy? Am J Roentgenol 2008; 190: $666-670$

20 Ho A et al. Adverse Events with Universal Use of Iodixanol for CT: Comparison With Iohexol. J Comput Assist Tomogr 2007; 2: 165-168

21 Katayama H, Yamaguchi K, Kozuka T et al. Adverse reactions to ionic and nonionic contrast media: a report from the Japanese committee on the safety of contrast media. Radiology 1990; 175: 621-628

22 Cochran ST, Bomyea K, Sayre JM. Trends in adverse events after IV administration of contrast media. Am J Roentgenol 2001; 176: 1385 1388

23 Mortelé KJ, Oliva MR, Ondategui S et al. Universal use of nonionic iodinated contrast medium for CT: evaluation of safety in a large urban teaching hospital. Am J Roentgenol 2005; 185: 31 - 34

24 McCullough PA. Contrast-Induced acute kidney injury. Journal of the American College of Cardiology 2008; 51: 1419-1428

25 Davidson CJ, Erdogan AK. Contrast Media: Procedural capacities and potential risks Reviews in cardiovascular medicine; 2008; 9: S24-S34 